\title{
Curva de crescimento de diferentes linhagens de frango de corte caipira
}

\author{
Growth curve of different strains of broiler chickens
}

\author{
Jonas Morais ${ }^{\mathrm{I}}$ Priscila Becker Ferreira ${ }^{\mathrm{II}}$ Ianglio Márcio Travassos Duarte Jacome ${ }^{\mathrm{III}}$ \\ Renius Mello ${ }^{\mathrm{IV}}$ Fernanda Cristina Bredav ${ }^{\mathrm{P}}$ Paulo Roberto Nogara Rorato $^{\mathrm{V}}$
}

\section{RESUMO}

Objetivou-se identificar o modelo de regressão não linear mais adequado para descrever a curva de crescimento de quatro linhagens de frangos caipira e compará-las entre as linhagens. Foram utilizadas 100 aves, sendo 13 machos e 12 fêmeas de cada uma das linhagens. Foram testados 13 modelos de regressão não linear e, após a seleção do melhor modelo, foi verificada a igualdade de parâmetros e identidade de modelos não lineares. $O$ peso médio aos 77 dias de idade foi de 3.017, 2.781, 2.724 e 2.587kg para a Pesadão, Mista, Carijó e Pescoço Pelado, respectivamente. O modelo Quadrático Logaritmico apresentou melhor ajuste dos dados para as linhagens Pesadão, Carijó e Mista, segundo o quadrado médio do resíduo e desvio médio absoluto. Para a linhagem Pescoço Pelado, os critérios estatísticos apontaram como mais adequado o modelo Polinomial Inverso, seguido do Quadrático Logarítmico. As curvas de crescimento entre machos e entre fêmeas das linhagens foram diferentes, assim, como as curvas de crescimento de machos e fêmeas dentro de linhagens. A divergência entre as classes avaliadas foi atribuida à taxa de decréscimo de peso, parâmetro c do modelo Quadrático Logarítmico. O modelo Quadrático Logarítmico pode ser utilizado para descrever a curva de crescimento das quatro linhagens. A linhagem Pesadão tem o crescimento mais acelerado e a linhagem Pescoço Pelado tem o crescimento mais lento. A partir do $35^{\circ}$ dia de idade, sugere-se o ajuste do manejo nutricional para alterar a curva de crescimento e atender a legislação vigente e o mercado consumidor.

Palavras-chave: quadrático logaritmico, peso corporal, regressão não linear.

\section{ABSTRACT}

This study aimed to identify the nonlinear regression model more adequate to describe and compare the growth curve of four strains of broiler chickens. One hundred birds were used, 13 males and 12 females from each strains. There were tested 13 models of nonlinear regression and after that the best model was used to test the parameters equality and the models identity. The average weight at 77 days of age was 3.017, 2.781, 2.724 and $2.587 \mathrm{~kg}$ to the strains Hulking, Mixed, Carijo and Naked Neck, respectively. The Quadratic Logarithmic model showed better fit the data from Hulking, Carijó and Mixed strains, according to the mean square and mean absolute deviation tests. To the Naked Neck line the statistical criteria showed as more suitable the Inverse Polynomial model followed by the Quadratic Logarithmic. The males and females growth curves were different between and within the strains. The divergence between the evaluated classes was attributed to the decrease of weight rate, $c$ parameter of Quadratic Logarithmic model. Quadratic Logarithmic model could be used to describe the growth curve of the four strains. The Hulking strain presented the faster and the Naked Neck strain presented the slowest growth. The feed system with the objective of change the growth curve must be adopted by the $35^{\text {th }}$ day of age, to attend the current legislation and the consumer market.

Key words: quadratic logarithmic, body weight, nonlinear regression.

\section{INTRODUÇÃO}

A criação de frangos para a produção de carne tipo caipira é um dos segmentos da avicultura alternativa que tem se mostrado promissor, pois, além de agregar valor ao produto e utilizar um sistema de criação que preza pelas normas de bem estar animal, serve tanto para pequenos e médios produtores como para a produção em escala comercial.

ICurso de Graduação em Zootecnia, Universidade Federal de Santa Maria (UFSM), Santa Maria, RS, Brasil.

"Programa de Pós-graduação em Zootecnia, Departamento de Zootecnia, UFSM, 97105-900, Santa Maria, RS, Brasil. E-mail: pri_zoot@hotmail.com. Autor para correspondência.

"IIDepartamento de Zootecnia e Ciências Biológicas, UFSM, Campus de Palmeiras das Missões, Palmeiras das Missões, RS, Brasil.

IV Departamento de Tecnologia e Ciência dos Alimentos, UFSM, Santa Maria, RS, Brasil.

vepartamento de Zootecnia, UFSM, Santa Maria, RS, Brasil. 
Apesar de as linhagens caipiras apresentarem menor potencial de crescimento, desempenho zootécnico e rendimento de partes nobres que os frangos de corte comerciais, sua criação é justificada por atributos diferenciados na qualidade da carne mais próxima da exigida pelo mercado consumidor, como textura e coloração da carne mais acentuada (SANTOS et al., 2005).

O conhecimento da curva de crescimento das diferentes linhagens de frango pode auxiliar na sua escolha, na adoção de práticas de manejo que otimizem a produção de carne, priorizando as necessidades nutricionais de cada fase de crescimento, assim estabelecendo programas alimentares específicos, bem como na definição da idade ótima de abate (BRACCINI NETO, 1993). Dentre os métodos existentes para estimar a curva de crescimento dos animais, os modelos não lineares têm sido desenvolvidos de forma a relacionar os dados de peso/idade e têm se mostrado adequados para descrever curvas de crescimento (OLIVEIRA et al., 2000).

Segundo SILVA et al. (2010), uma vantagem dos modelos não lineares é a possibilidade de interpretação biológica dos parâmetros, que é um importante critério na avaliação dos modelos de curva de crescimento. Isso permite um maior entendimento do processo de crescimento dos animais. Assim, as curvas de crescimento permitem resumir em três ou quatro parâmetros as características de crescimento da população (peso inicial, velocidade de crescimento e peso adulto).

Objetivou-se identificar, por meio de diferentes critérios estatísticos, o modelo de regressão não linear mais adequado para descrever a curva de crescimento de quatro linhagens de frangos caipira para produção de carne e comparar as diferentes linhagens sobre o mesmo manejo.

\section{MATERIAL E MÉTODOS}

Os animais foram criados no Laboratório de Avicultura do Departamento de Zootecnia e Ciências Biológicas da Universidade Federal de Santa Maria, campus de Palmeiras das Missões, RS, durante os meses de setembro a novembro de 2010. Inicialmente, foram utilizadas 100 aves, sendo 13 machos e 12 fêmeas de cada uma das linhagens avaliadas: Pesadão, Pescoço Pelado, Carijó e Mista. Com um dia de idade, foi feita a identificação (anilhas) e pesagem individual dos pintos.

Água e ração foram disponibilizadas à vontade durante todo o período experimental. Foi adotado um programa de alimentação em duas fases, como segue: inicial ( $1^{\circ}$ ao $28^{\circ}$ dia de idade), na qual foi fornecida dieta com $40 \%$ de concentrado e $60 \%$ milho; e crescimento ( $29^{\circ}$ ao $77^{\circ}$ dia de idade), com $35 \%$ de concentrado e $65 \%$ de milho.

O concentrado comercial utilizado era composto por: ácido fítico, calcário calcítico, cloreto de colina, cloreto de sódio, farelo de soja, farelo de trigo, farinha de vísceras, farinha de carne e ossos bovinos, farinha de vísceras de aves, hidróxido tolueno butilado (B.H.T), iodato de cálcio, monensina sódica, niacina, pantotenaxo de cálcio, selenito de sódio, sulfato de cobre, sulfato de ferro, sulfato de manganes, sulfato de zinco e vitaminas A/D3, B1, B12, B2, B6, D3, E e K3. Tendo na dieta fornecida (milho mais concentrado) os seguintes níveis nutricionais calculados: $18,50 \%$ de proteína bruta, 3,202Mcal kg-1 de energia metabolizável, $0,161 \%$ de cálcio, $0,368 \%$ de fósforo disponível, $0,436 \%$ de metionina, $1,060 \%$ de lisina, $0,742 \%$ de metionina mais cistina, $0,811 \%$ de treonina, $0,279 \%$ de triptofano e $0,193 \%$ de sódio.

Após $29^{\circ}$ dia de idade, as aves foram criadas em um sistema semiconfinado, no qual os animais tiveram acesso a uma área verde de campo nativo (área: $25 \times 25 \mathrm{~m}=$ $625 \mathrm{~m}^{2}$ ). Todas as aves foram vacinadas contra as doenças de New Castle, Gumboro, Broquite H120 e Bouba.

Os animais foram pesados a cada sete dias, sendo excluídas das análises aves com menos de quatro registros. Portanto, foram excluídas informações de quatro aves da linhagem Mista (três fêmeas e um macho), pois estas morreram antes de completar o mínimo de quatro registros. $\mathrm{O}$ arquivo final continha 1043 registros de peso de 96 aves.

Para o ajuste dos modelos de regressão não linear, foram testados os 13 seguintes modelos: Brody I (BRODY et al., 1923), Brody II (BRODY et al., 1924), Brody III (BRODY, 1945), Cobuci (COBUCI et al., 2000), Polinomial Inverso (NELDER, 1966), Quadrático Logarítmico (BIANCHINI SOBRINHO, 1984), Papajcsik e Bodero (PAPAJCSIK \& BODERO, 1988), Logístico (NELDER, 1961), Richards (RICHARDS, 1959), Von Bertalanfy (VON BERTALANFY, 1957), Gompertz (LAIRD, 1966), Função Gama Incompleto (WOOD, 1967) e Linear Hiperbólico (BIANCHINI SOBRINHO, 1984). Os parâmetros dos modelos foram estimados pelo método de Gauss Newton modificado, que se baseia numa aproximação por uma série de Taylor de primeira ordem para produzir uma linearização da função não linear (REGAZZI, 2003), utilizando o procedimento NLIN do programa estatístico SAS ${ }^{\circledR}$ (2001).

Para a escolha da função que melhor descreve a curva de crescimento, foram utilizados os seguintes critérios estatísticos: quadrado médio do resíduo (QMR), calculado dividindo-se a soma de quadrados do resíduo pelo número de observações, o qual é o estimador da máxima verossimilhança da variância residual, para que se possa comparar o QMR dos diferentes modelos, pois possuem número diferente de parâmetros a serem estimados; e o desvio médio absoluto dos resíduos (DMA), estatística proposta por SARMENTO et al. (2006), 
utilizada para avaliar a qualidade do ajuste, calculado conforme a fórmula a seguir: DMA $=\frac{\sum_{i=1}^{n}\left|Y_{i} \cdot \hat{Y}_{i}\right|}{\text {, }}$ sendo que: $Y i$ é o valor observado; $\hat{Y} i$ o valor estimado; $n$ o tamanho da amostra. Visualmente, foi analisada a distribuição dos resíduos e as curvas de crescimento observadas e estimadas. Os resultados de cada critério de seleção de modelos foram expressos por meio da comparação entre eles, uma vez que menores valores de QMR e DMA são preferidos.

Após a seleção do modelo, foi verificada a igualdade de parâmetros e identidade de modelos não lineares para determinar se uma única curva seria adequada em descrever o crescimento de aves das linhagens Pescoço Pelado, Pesadão, Carijó e Mista por sexo; e comparar as curvas de crescimento de machos e fêmeas dentro da mesma linhagem. Para este fim, aplicouse o teste da razão de verossimilhança com aproximações dadas pela estatística $F$ (REGAZZI \& SILVA, 2004).
Pelo teste de razão de verossimilhança, foram avaliadas 15 hipóteses e, para verificar a melhor dentre as aceitas, foi utilizado o critério de informação de Akaike - AIC (AKAIKE, 1974) e o critério de informação Bayesiano - BIC (SCHWARZ, 1978), obtidos da seguinte forma: $B I C=-2 L(\tilde{\theta})+\ln (N) d$ ; em que: $d$ representa o número total de parâmetros estimados pelo modelo e $N$ é o número total de observações e o logaritmo de verossimilhança restrita.

\section{RESULTADOS E DISCUSSÃO}

A divergência no crescimento das aves das diferentes linhagens começa a ser observada a partir do $35^{\circ}$ dia de idade, sendo a maior diferença verificada entre as linhagens Pesadão e Pescoço Pelado e a menor entre as linhagens Carijó e Mista (Figura 1a). O peso médio aos 77 dias de idade foi de 3.017, 2.781, $2.724 \mathrm{e}$

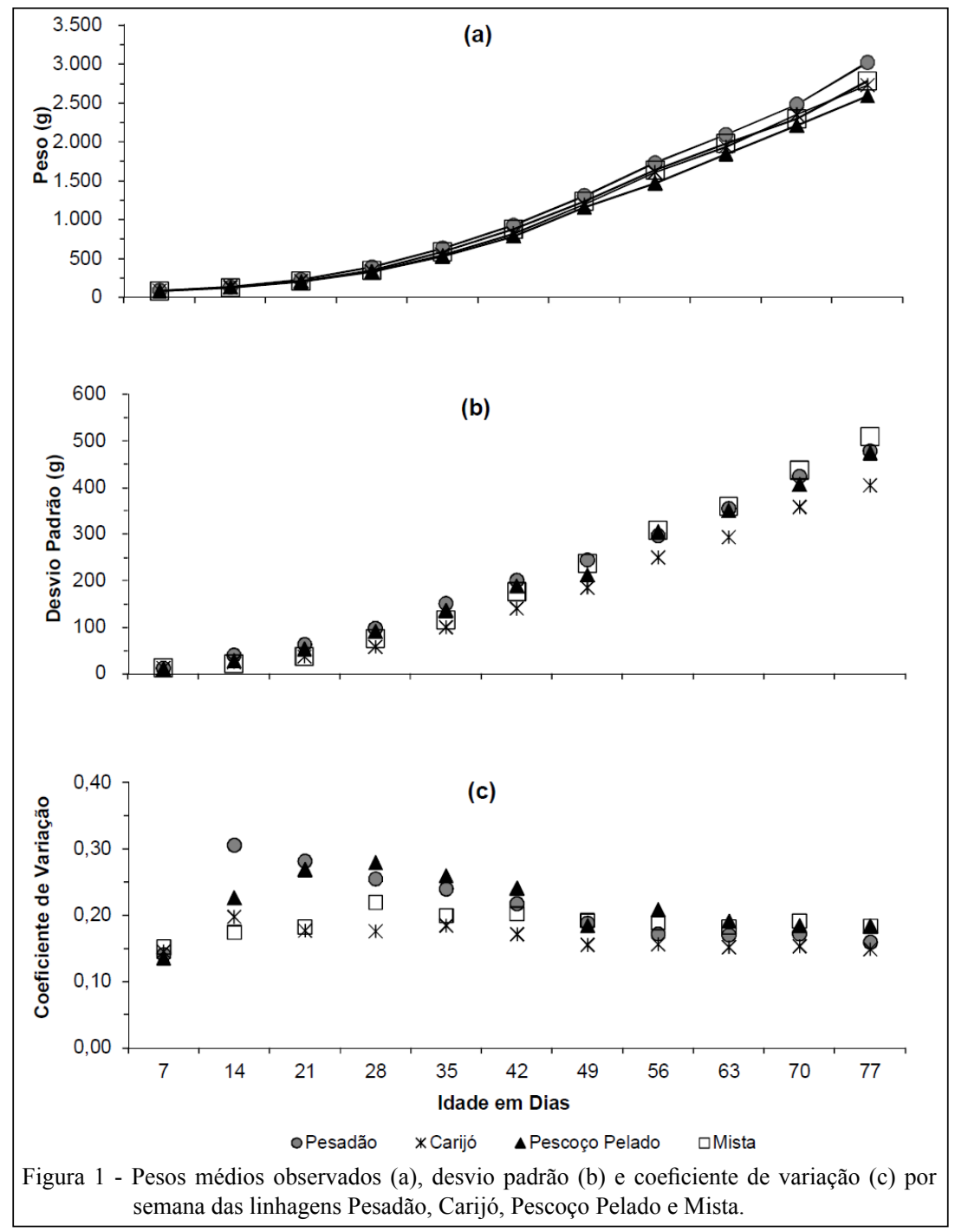

Ciência Rural, v.45, n.10, out, 2015. 
2.587kg para a Pesadão, Mista, Carijó e Pescoço Pelado, respectivamente. A linhagem Pesadão mostrou menor ganho de peso no 56으 dia (365g vs 376g Pescoço Pelado) e $63^{\circ}$ dia (387g vs $411 \mathrm{~g}$ Carijó).

A linhagem Pescoço Pelado foi a que apresentou o crescimento mais lento. De acordo com FONSECA et al. (2010), as aves Pescoço Pelado apresentam melhores mecanismos de dissipação do calor, o que favorece a manutenção do conforto térmico no verão. SILVA et al. (2003) relataram que o frango caipira Pescoço Pelado é o que melhor se adapta ao clima brasileiro e apresenta um bom desempenho. Nesse sentido, observou-se que, dos 42 aos 77 dias de idade, esta linhagem apresentou ganho de peso praticamente constante ( $370 \mathrm{~g})$, enquanto, nas demais linhagens, este comportamento não foi observado. Isso pode ser explicado pela melhor adaptabilidade dessa linhagem ao clima da região Noroeste do Rio Grande do Sul.

De acordo com o MAPA (1999), Ofício circular DOI/DIPOAno 007/99 de 19/05/1999, na criação de frangos caipiras, as aves devem ser abatidas com, no mínimo, 85 dias de idade, com aproximadamente $2,5 \mathrm{~kg}$. Portanto, as linhagens e o manejo alimentar utilizados neste trabalho não foram adequados para atender a legislação vigente e o mercado consumidor, já que as aves foram abatidas com 77 dias, pois já haviam atingido o peso desejado (peso superior a 2,5kg). Assim, todas as linhagens devem ser acompanhadas de práticas de manejo alimentar, visando à modificação da curva de crescimento, reduzindo a velocidade de ganho de peso, associado ao uso de linhagem de crescimento lento, por exemplo, a Pescoço Pelado.

Para todas as linhagens, o comportamento do desvio padrão ao longo da trajetória do crescimento foi crescente e variou de 11,45 a 478,17g para a Pesadão, 11,52 a 403,85g para a Carijó, 10,18 a 473,30g para a Pescoço Pelado e de 11,66 a 508g para a Mista. Até o $49^{\circ}$ dia de idade, as linhagens apresentaram estimativas de desvio padrão semelhantes, após, a linhagem Carijó resultou em desvios padrão inferiores (Figura 1b).

O coeficiente de variação é interpretado como a variabilidade dos dados em relação à média, ou seja, quanto menor o coeficiente de variação, mais homogêneo o conjunto de dados (PIMENTEL-GOMES, 1987). Um coeficiente de variação é considerado baixo quando for menor ou igual a 0,25 , o que não ocorreu nas idades $14(0,30)$ e $21(0,28)$ dias para a linhagem Pesadão e $21(0,27), 28(0,28)$ e $35(0,26)$ dias para a linhagem Pescoço Pelado (Figura 1c).

Na tabela 1, estão os resultados dos critérios estatísticos de seleção de modelos que convergiram para as diferentes linhagens. O modelo Quadrático Logaritmo apresentou melhor ajuste dos dados para as linhagens Pesadão, Carijó e Mista, segundo o QMR e DMA. Para a linhagem Pescoço Pelado, os critérios estatísticos apontaram como mais adequado o modelo Polinomial Inverso, seguido do Quadrático Logarítmico.

Tabela 1 - Estimativa dos parâmetros, número de iterações (NI), quadrado médio do resíduo (QMR) e desvio médio absoluto (DMA) dos modelos que convergiram para as diferentes linhagens.

\begin{tabular}{|c|c|c|c|c|c|}
\hline Linhagens & Modelo* & Parâmetros Estimados & $\mathrm{NI}$ & QMR & DMA \\
\hline \multirow{3}{*}{ Pesadão } & Brody $\mathrm{I}^{1}$ & $Y t=193,8$ exp- $0,0364 t$ & 39 & 90937 & 140,39 \\
\hline & Papajasik e Bodero $^{2}$ & $Y t=11,21 t \exp -0,016 t$ & 08 & 72555 & 71,57 \\
\hline & Quadrático Logarítmico $^{3}$ & $\mathrm{Yt}=562,2+25,79 \mathrm{t}+0,33 \mathrm{t}^{2}-340 \ln \mathrm{t}+\varepsilon$ & 01 & 67312 & 26,62 \\
\hline \multirow{4}{*}{ Carijó } & Brody I & $Y t=177,8$ exp- $0,0364 t$ & 23 & 69649 & 144,49 \\
\hline & Polinomial Inverso ${ }^{4}$ & $\mathrm{Yt}=\left(0,00508-0,00012+8,24 \mathrm{E}-07 \mathrm{t}^{2}\right)^{-1}+\varepsilon$ & 24 & 59836 & 112,4 \\
\hline & Papajasik e Bodero & $Y t=10,2 t \exp -0,016 t$ & 10 & 53113 & 81,82 \\
\hline & Quadrático Logarítmico & $\mathrm{Yt}=681,4+29,61 \mathrm{t}+0,26 \mathrm{t}^{2}-408,9 \ln \mathrm{t}+\varepsilon$ & 1 & 46734 & 37,94 \\
\hline \multirow{4}{*}{ Pescoço Pelado } & Brody I & $Y t=167,88 \exp -0,03 t$ & 28 & 81583 & 130,22 \\
\hline & Polinomial Inverso & $\mathrm{Yt}=\left(0,14-0,002+0,00001 \mathrm{t}^{2}\right)^{-1}+\varepsilon$ & 14 & 62877 & 27,63 \\
\hline & Papajasik e Bodero & $Y t=9,67 t \exp -0,01 t$ & 9 & 67533 & 69,85 \\
\hline & Quadrático Logarítmico & $\mathrm{Yt}=555+24,02 \mathrm{t}+0,27 \mathrm{t}^{2}+-329,4 \ln \mathrm{t}+\varepsilon$ & 1 & 63001 & 29,25 \\
\hline \multirow{3}{*}{ Mista } & Brody I & $Y t=186,3 \exp -0,03 t$ & 27 & 93826 & 143,72 \\
\hline & Papajasik e Bodero & $Y t=10,82 t \exp -0,01 t$ & 8 & 76185 & 78,88 \\
\hline & Quadrático Logarítmico & $\mathrm{Yt}=695,9+34,12 \mathrm{t}+0,22 \mathrm{t}^{2}-435,8 \ln \mathrm{t}+\varepsilon$ & 1 & 70166 & 33,79 \\
\hline
\end{tabular}

${ }^{1}$ BRODY et al. (1923): Yt=ae(-ct) $+\varepsilon ;{ }^{2}$ PAPAJCSIK \& BODERO (1988): Yt $=$ at ${ }^{*}$ exp-ct $+\varepsilon ;{ }^{3}$ BIANCHINI SOBRINHO (1984): Yt $=\mathrm{a}+$ $\mathrm{bt}+\mathrm{ct}^{2}+\mathrm{d} \ln (\mathrm{t})+\varepsilon .{ }^{4} \mathrm{NELDER}(1966): \mathrm{Yt}=\mathrm{t} /(\mathrm{a}+\mathrm{bt}+\mathrm{ct} 2)+\varepsilon$. Interpretações dos parâmetros dos modelos: Yt é peso corporal em $\mathrm{t}$ semanas de idade; a é o valor assintótico do peso; b a taxa de aumento do peso; c a taxa de decréscimo do peso; d não possui interpretação biológica. 
$\mathrm{Na}$ literatura, observa-se que, para frangos comerciais, os modelos que apresentam melhor ajuste para descrever o crescimento de frangos de corte de linhagens comerciais são Gompertz, Logístico, Von Bertalanffy e Brody I, derivados da curva Richards (MIGNONGRASTEAU \& BEAUMONT, 2000).

$\mathrm{Na}$ visualização gráfica dos dados observados e estimados pelos modelos (dados não apresentados), observou-se que o modelo Quadrático Logarítmico ajustou de forma adequada o peso das aves da linhagem Pescoço Pelado, assim, optou-se para as demais análises por este modelo. O modelo Quadrático Logarítmico, descrito por BIANCHINI SOBRINHO (1984), é dado pela equação $\mathrm{Yt}=\mathrm{a}+$ $\mathrm{bt}+\mathrm{ct}^{2}+\mathrm{d} \ln (\mathrm{t})+\varepsilon$ em que: a é o valor assintótico do peso; b a taxa de aumento do peso; c a taxa de decréscimo do peso; e d não possui interpretação biológica.

Após a escolha do melhor modelo para descrever a trajetória de crescimento, aplicou-se o teste de identidade para identificar as semelhanças e diferenças entre as curvas de crescimento de machos de diferentes linhagens e de fêmeas de diferentes linhagens.

A hipótese que testou a identidade de modelos foi rejeitada, ou seja, os machos das diferentes linhagens possuem curvas diferentes. Assim, as análises devem ser processadas utilizando o modelo quadrático logarítmico, todavia, para cada linhagem, deve ser processada uma análise distinta. $\mathrm{O}$ mesmo ocorreu para as fêmeas.

Procedeu-se à igualdade de parâmetros e verificou-se que 10 hipóteses foram aceitas comparando os machos das diferentes linhagens e 14 aceitas para as fêmeas de diferentes linhagens. O AIC e BIC apontaram como a melhor hipótese para os machos a $\mathrm{H}_{01}$, que considera os parâmetros a, b e d iguais para todas as linhagens. Assim, para descrever a curva de crescimento dos machos, apenas o parâmetro c do modelo quadrático logarítmico é diferente para as quatro linhagens.

Os machos das diferentes linhagens apresentaram crescimento semelhante até os 35 dias de idade. A partir dessa idade, os machos da linhagem Pesadão começaram a ganhar mais peso que as demais, chegando aos 77 dias de idade com $3.427 \mathrm{~kg}$. Os machos da linhagem Pescoço Pelado, a partir dos 35 dias, ganharam menos peso que as demais, alcançando, aos 77 dias de idade, $2.964 \mathrm{~kg}$. Os machos das linhagens Carijó e Mista apresentaram crescimento semelhante e intermediário.
Com relação às fêmeas, o AIC apontou as hipóteses $\mathrm{H}_{01}=$ os parâmetros $\mathrm{a}, \mathrm{b}$ e d são iguais e $\mathrm{H}_{02}=$ os parâmetros a, c e d são iguais; e o BIC as hipóteses $\mathrm{H}_{01}, \mathrm{H}_{02}$ e $\mathrm{H}_{03}$ em que os parâmetros c e d são iguais para todas as linhagens. Ao plotar a curva observada e estimada, considerando as três hipóteses (dados não apresentados), observou-se que os três modelos ajustaram a curva de crescimento de forma satisfatória para as quatro linhagens. Todavia, optouse pela $\mathrm{H}_{01}$, por ser o mesmo apontado na comparação entre machos e por apresentar menor número de parâmetros a serem estimados.

Para identificar as semelhanças e diferenças entre as curvas de crescimento de machos e fêmeas dentro de linhagem, observou-se que a hipótese que testa a identidade de modelos foi rejeitada para todas as linhagens. Esse resultado indica que, dentro de linhagem, machos e fêmeas possuem curvas diferentes (Figura 2).

Com relação às hipóteses de igualdade de parâmetros, observou-se que todas foram aceitas para a linhagem Pesadão, 10 foram aceitas para a linhagem Carijó, 12 para a linhagem Pescoço Pelado e 9 para a Mista. O AIC e BIC apontaram H01 como a melhor hipótese para descrever a curva de machos e fêmeas da linhagem Carijó, Pescoço Pelado, Mista e Peladão.

Na figura 2, são apresentados os pesos observados e estimados pelo modelo Quadrático Logarítmico, hipótese H01, para as quatro linhagens. Em todas as linhagens verificou-se ganho de peso semelhante entre machos e fêmeas nas idades iniciais. Divergências entre os sexos foram verificadas após os 35 dias nas linhagens Pesadão e Carijó e após os 42 dias nas linhagens Pescoço Pelado e Mista, sendo as maiores diferenças entre os sexos observadas na linhagem Pesadão (700g aos 77 dias) e as menores na linhagem Pescoço Pelado (324g aos 77 dias).

\section{CONCLUSÃO}

O modelo Quadrático Logarítmico pode ser utilizado para descrever a curva de crescimento das linhagens Pesadão, Carijó, Mista e Pescoço Pelado, porém o parâmetro c do modelo é diferente para as quatro linhagens avaliadas e para machos e fêmeas.

A partir do $35^{\circ}$ dia de idade, sugere-se que se ajuste o manejo nutricional para alterar a curva de crescimento e atender à legislação vigente e o mercado consumidor. Levando-se em consideração que a linhagem Pesadão tem o crescimento mais acelerado e a linhagem Pescoço Pelado tem o crescimento mais lento. 


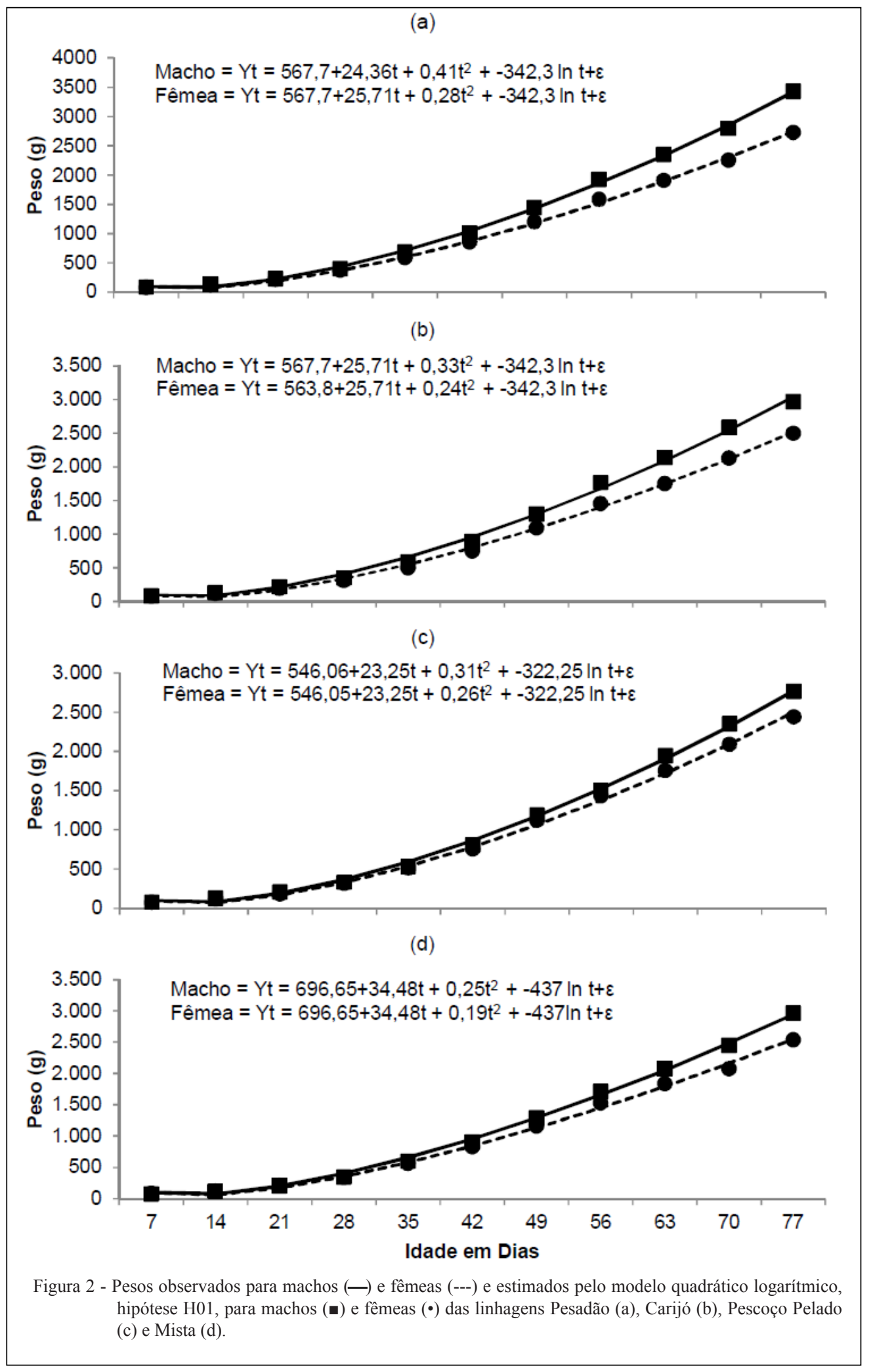

Ciência Rural, v.45, n.10, out, 2015. 


\section{REFERÊNCIAS}

AKAIKE, H. A new look at the statistical model identification. IEEE Transactions on Automatic Control, Boston, v.19, n.6, p.716-723, 1974.

BIANCHINI SOBRINHO, E. Estudo da curva de lactação de vacas da raça Gir. 1984. 88f. Tese (Doutorado em Genética) Faculdade de Medicina Veterinária de Ribeirão Preto, Universidade de São Paulo, SP.

BRACCINI NETO, J. Estudo genético de curvas de crescimento de aves de postura. 1993. 102f. Dissertação (Mestrado em Ciências) - Curso de Pós-graduação em Zootecnia, Universidade Federal de Pelotas, RS.

BRODY, S.A. et al. The rate of decline of milk secretion with the advance of the period of lactation. Journal of General Physiology, v.5, p.441-444, 1923.

BRODY, S.A. et al. The relation between the initial rise and the subsequent decline of milk secretion following parturition. Journal of Genetic Physiology, v.6, p.541-545, 1924.

BRODY, S. Bioenergetics and growth. New York: Rheinhold, 1945. 645p.

COBUCI, J.A. et al. Curva de Lactação na Raça Guzerá. Revista Brasileira de Zootecnia, v.29, n.5, p.1329-1332, 2000. Disponível em: <http://www.scielo.br/scielo.php?pid=S1516$35982000000500011 \&$ script $=$ sci arttext $>$. Acesso em: $12 \mathrm{dez}$. 2012. doi:10.1590/S1516-35982000000500011.

FONSECA, R.A. et al. Avaliação de linhagens de aves de corte tipo Caipira submetidas ao bioclima do litoral do Paraná. Scientia Rural, p.49-63, 1 a ed, 2010. Disponível em: <http:// www.cescage.edu.br/site/pagina/arquivos/revista/innovare/ artigos/2b1davaliacao de linhagens de aves de corte tipo caipira_submetidas_ao_bioclima_do_litoral_do_parana.pdf $>$. Acesso em: 12 out. 2013 .

LAIRD, A.K. Dynamics of relative growth. Bar Habor, v.29, p.249-263, 1966.

MIGNON-GRASTEAU, S. et al. Genetic analysis of growth curve parameters for male and female chickens resulting from selection on shape of growth curve. Journal Animal Science, v.78, p.2515$2524,2000$.

MINISTÉRIO DA AGRICULTURA, PECUÁRIA E DO ABASTECIMENTO (MAPA). DEPARTAMENTO DE INSPEÇÃO DE PRODUTOS DE ORIGEM ANIMAL (DIPOA). DIVISÃO DE OPERAÇÕES INDUSTRIAIS (DOI). Ofício Circular DOI/DIPOA n. 007/99. Registro do produto "Frango Caipira" ou "Frango Colonial" ou "Frango Tipo ou Estilo Caipira" ou "Tipo ou Estilo Colonial". 19/05/1999. Online. Disponível em: <http://www.uov.com.br/biblioteca/158/normas para_a_criacao_de_frango_e_galinha_caipira.html $>$. Acesso em: 23 mar. 2013.

NELDER, J.A. The fitting of a generalization of the logistic curve. Biometrics, v.17, p.89-94, 1961.

NELDER, J.A. Inverse polynomials a useful group of multi-factor response functions. Biometrics, v.22, n.1, p.128-141, 1966.
OLIVEIRA, H.N. et al. Comparação de modelos não lineares para descrever o crescimento de fêmeas da raça Guzerá. Pesquisa Agropecuária Brasileira, v.35, p.1843-1851, 2000. Disponível em: <http://base.repositorio.unesp.br/ bitstream/handle/11449/27322/S0100-204X2000000900017. pdf? sequence=1\&isAllowed=y>. Acesso em: 15 abr. 2014. doi:10.1590/S0100-204X2000000900017.

PAPAJCSIK, I.A.; BODERO, J. Modelling lactation curves of Friesian cows in a subtropical climate. Animal Production, v.47, p.201-207, 1988. Disponível em: <http://journals.cambridge.org/ action $/$ displayAbstract fromPage $=$ online $\&$ aid $=7380176 \&$ fileId $=$ S0003356100003275 $>$. Acesso em: 12 set. 2013. doi:10.1017/ S0003356100003275.

PIMENTEL-GOMES, F. Curso de estatística experimental. 12.ed. Piracicaba: Livraria Nobel, 1987. 467p.

REGAZZI, A.J. Teste para verificar igualdade de parâmetros e a identidade de modelos de regressão não linear. Revista CERES, v.50, n.287, p.9-26, 2003.

REGAZZI, A.J.; SILVA, C.H.O. Teste para verificar a igualdade de parâmetros e a identidade de modelos de regressão não linear (Dados no delineamento inteiramente casualizado). Revista de Matemática e Estatística, v.22, n.3, p.33-45, 2004.

RICHARDS, F.J. A flexible growth function for empirical use. Journal of Experimental Botany, v.10, p.290-300, 1959.

SANTOS, A.L. et al. Estudos do crescimento, desempenho, rendimento de carcaça e qualidade de carne de três linhagens de frango de corte. Revista Brasileira de Zootecnia, v.34, n.5, p.1589-1598, 2005. Disponível em: <http://www.scielo. $\mathrm{br} / \mathrm{scielo} . \mathrm{php}$ ? pid $=\mathrm{S} 1516-35982005000500020 \& \mathrm{script}=\mathrm{sci}$ arttext>. Acesso em: 13 jan. 2013. doi:10.1590/S151635982005000500020 .

SARMENTO, J.L. et al. Estudo da curva de crescimento de ovinos Santa Inês. Revista Brasileira de Zootecnia, v.35, n.2, p.435-442, 2006. Disponível em: <http://www.scielo.br/scielo. php? script $=$ sci_arttext\&pid=S1516-35982006000200014>. Acesso em: 15 mar. 2013.

SAS (STATISTICAL ANALYSES SYSTEM). User's guide: Stat, Version 8 ed. Cary: SAS Institute, USA, 2001, 1464p.

SCHWARZ, G. Estimating the dimensional of a model. Annals of Statistics, v.6, n.2, p.461-464, 1978.

SILVA, M.J. et al. Avicultura alternativa como fonte de renda e melhoria na qualidade de vida nas propriedades de produção familiar. Juiz de Fora: Anais da SOBER, 2003. p.146.

SILVA, N.A.M. et al. Modelo hierárquico Bayesiano aplicado na avaliação genética de curvas de crescimento de bovinos de corte. Arquivo Brasileiro de Medicina Veterinária e Zootecnia, v.62, p.647-654, 2010. Disponível em: <http://www.scielo.br/pdf/ abmvz/v62n2/22.pdf>. Acesso em: 15 jun. 2013. doi: 10.1590/ S0102-09352010000200022.

VON BERTALANFFY, L. Quantitative laws in metabolism and growth. Quarterly Review of Biology, v.32, p.218, 1957.

WOOD, P.D.P. Algebraic model of the lactation curve in cattle. Nature, n.216, p.164-165, 1967. 